\title{
Electrospinning de fibras submicrométricas de Fibroína de seda con Sangre de drago (Croton Lechleri Müll) para la producción de apósitos
}

\author{
Jorge Gabriel Melo ${ }^{\mathrm{a}}$, Nathaly Villacís ${ }^{\mathrm{a}}$, Karla Vizuete ${ }^{\mathrm{b}}$, Carlos Arroyo ${ }^{\mathrm{b}}$, Christian Narváez ${ }^{\mathrm{a}}$ \\ ${ }^{a}$ Departamento de Ciencias de la Energía y Mecánica, Universidad de las Fuerzas Armadas ESPE \\ ${ }^{\mathrm{b}}$ Centro de Nanociencia y Nanotecnología, Universidad de las Fuerzas Armadas ESPE \\ jgmelo@espe.edu.ec, cnvillacis@espe.edu.ec, ksvizuete@espe.edu.ec, cpnarvaez1@espe.edu.ec, ccarroyo@espe.edu.ec
}

\begin{abstract}
Resumen-El presente trabajo de investigación hace uso de la técnica de electrospinning. Los biomateriales utilizados fueron fibroína de seda que resalta entre otros biopolímeros por sus excelentes propiedades mecánicas y biológicas dopado con sangre de drago, savia natural con muchas bondades curativas utilizadas ancestralmente por pueblos indígenas de la Amazonía. Tras la extracción del biopolímero y la preparación de la solución acuosa usando óxido de polietileno (PEO) de 1000 [KDa]. Las muestras fueron caracterizadas de las siguientes formas: morfológicamente a través de microscopía SEM (microscopio electrónico de barrido) obteniendo diámetros entre 139,43 - 160 [nm] y mecánicamente en microscopía de fuerza atómica (AFM) obteniendo módulos de Young de 279,9[MPa] sin sangre de drago y 107,1 [MPa] con sangre de drago.
\end{abstract}

Palabras Claves-Apósitos, Electrospinning, Fibroína de Seda, Sangre de drago.

\begin{abstract}
This research work makes use of the electrospinning technique. The biomaterials used were silk fibroin that stand out among others, biopolymers for their natural mechanical and biological properties, with drago's blood, natural sap with many healing benefits, used ancestrally by indigenous peoples of the Amazon. After the extraction of the biopolymer and the preparation for the production of polyethylene oxide (PEO) of 1000 [KDa]. The samples were characterized in the following ways: morphologically through SEM microscopy (scanning electron microscope) obtaining the parameters between $139.43-160$ [nm] and mechanically in atomic force microscopy (AFM) obtaining Young's modules of 279.9 [MPa] without drago's blood and 107.1 [MPa] with drago's blood.
\end{abstract}

Keywords - Wound dressings, Electrospinning, Silk Fibroin, Drago's blood.

\section{INTRODUCCIÓN}

La técnica de electrospinning permite la formación de fibras poliméricas a escala nano y micrométricas mediante el uso fuerzas electrostáticas, los mismos que presentan características particulares entre las que resaltan: alta relación área/volumen, flexibilidad, alta porosidad y un rendimiento mecánico relativamente superior comparados con otros tipos de material de características morfológicas similares; así como la posibilidad de combinar las propiedades de varios polímeros para la formación de fibras [1].

Las fibras obtenidas con el tiempo han encontrado cabida en una serie de aplicaciones biomédicas antes desconocidas entre las cuales resaltan: andamios de tejidos [2], apósitos [3]. Los apósitos o wound dressing son elementos que ayudan a evitar la infección y mantener un entorno apropiado que permita la correcta cicatrización de la herida [4] para ello debe contar con ciertas características esenciales como la capacidad hemostática, que son los mecanismos que detienen las hemorragias, poder antibacteriano, absorción de excesos de líquidos exudados como fluidos de la herida o pus, transmisión apropiada entre agua y vapor, capacidad de ajustarse al contorno de la herida, adhesión funcional, es decir, que se adhiera al tejido sano, no al herido, indoloro para el paciente, capacidad de removerse con facilidad y finalmente que sea de bajo costo [5]-[8], esto fortalecido además por beneficios propios de la técnica como la facilidad de trabajar con una serie de polímeros biocompatibles, alta permeabilidad al oxígeno, tamaño de poro variable, la posibilidad de incorporar una variedad de moléculas bioactivas (fármacos como antimicrobianos, antiinflamatorios) en su estructura [9], [10] los pequeños diámetros de fibra, que se asemejan a la matriz extracelular (EM) y al área de superficie grande de una fibra que permite unir las proteínas y los receptores de la membrana celular durante el proceso de cultivo celular. Por lo tanto, son capaces de potenciar la proliferación celular y la formación de fibroplastos [11]- [13].

La fibroína de seda es el mayor constituyente proteico en los capullos del gusano de seda (alrededor del 72-81\%). En la actualidad se usa ampliamente como biomaterial en el campo biomédico, especialmente en ingeniería de tejidos y en medicina regenerativa, dado que facilita la adhesión de las células, estimula su crecimiento y permite la diferenciación, además, es biocompatible, resistente y biodegradable en fases controlables. [14], [15].

Además, presenta una serie de propiedades que la hacen muy interesante como biopolímero: es un material de naturaleza proteica, altamente biocompatible, no presenta rechazo ni respuesta inflamatoria en los tejidos donde se injerta [16]. El rechazo ocasional es producto de la contaminación de la fibroína con sericina generado por errores en el proceso de desgomado [17]. Presenta una gran resistencia mecánica a tracción, junto a una gran flexibilidad, es biodegradable, pero a un ritmo más lento que en otros biopolímeros orgánicos, lo que permite una mejor consolidación de los tejidos. En 2 años se reabsorbe totalmente y no se encuentran rastros de la misma en los tejidos [18]- [21].

Para ser electrohilable la solución acuosa de fibroína de seda es necesaria la mezcla con un polímero de alto peso molecular como el óxido de polietileno (PEO) que le brinde mejores propiedades reológicas, mayor viscosidad y 
concentración, parámetros muy importantes en el electrospinning. Para la ingeniería de tejidos dérmicos, la fibroína de seda tiene el potencial de actuar como un sustituto del colágeno, proporcionando soporte estructural y resistencia mecánica al injerto de ingeniería de tejidos [22].

La sangre de drago o croton lechleri Müll. es un árbol amazónico de pequeña envergadura común en las laderas andinas orientales de Perú, Colombia, Bolivia y Ecuador, cuya savia se ha utilizado históricamente sobre heridas para detener la hemorragia, acelerar el proceso de cicatrización evitando dejar huellas visibles o queloideas, sellar y proteger las lesiones de la infección. La savia se seca rápidamente y genera una barrera de tono rojizo, formando una "segunda piel" [23].

Su componente activo principal llamado taspina es un alcaloide encargado de la formación del colágeno y por ende la aceleración de la cicatrización, además de ser un excelente antibacteriano y antiséptico [24]- [28].

En el siguiente artículo se describe tanto el protocolo de preparación de extracción de la fibroína de seda como de la preparación de la solución acuosa, así como los resultados obtenidos de la caracterización de las membranas desarrolladas. En la sección II se presenta los materiales y métodos utilizados en la experimentación, en la sección III se presenta el análisis de resultados y finalmente, las conclusiones más relevantes son presentadas en la sección IV.

\section{Materiales Y MÉtodos}

\section{A. Materiales y Equipos}

Producto del proceso de experimentación se usó como materiales Sangre de drago orgánica certificada de la Fundación Chankuap de la ciudad de Macas, Ecuador, capullos de seda Bombyx Mori de la empresa Bao Jian Zhen y Óxido de polietileno (PEO) de 1000KDa S3 Chemical, mientras que para la extracción química se usó Carbonato de Sodio pureza del 99\% del laboratorio Panreac y Bromuro de litio pureza del 99\% del laboratorio S3 Chemical.

Además, para la preparación de las muestras se usó un agitador magnético Thermo Scientific S130815, una plancha de calentamiento y agitación BIPEE, un conductivímetro OHAUS Starter 300C y una balanza analítica Shimadzu TW423L de apreciación 0.01g.

\section{B. Preparación de la solución acuosa}

Dada la complejidad de manejar proteínas de alto peso molecular como la fibroína de seda es necesario la formulación de una solución acuosa reconstituida o regenerada o conocida como RSF (reconstituted silk fibroin) mediante un protocolo químico.

El protocolo químico usado centra su desarrollo en 3 puntos. El desgomado, hace uso de una solución salina de carbonato de sodio $\left(\mathrm{Na}_{2} \mathrm{CO}_{3}\right)$ 0.02M para la eliminación de la sericina, proteína de menor concentración porcentual en el capullo que une las fibras de fibroína, obteniendo finalmente fibroína pura en forma de una gasa o algodón. La disolución, se realizó con una solución salina de bromuro de litio( $\mathrm{LiBr}$ ) 9.3M durante 3 horas hasta que los filamentos de fibroína de seda se disuelvan en una solución de tonalidad ámbar altamente viscosa y conductora, propiedad nociva para el proceso de electrospinning. Por último, se realizó durante 3 días el proceso de diálisis que a través de continuos cambios de agua ultrapura permite realizar un intercambio de iones entre agua y la solución previamente cargada por el $\mathrm{LiBr}$ mediante el uso de membranas porosas de diálisis obteniendo finalmente la solución acuosa con adecuada conductividad eléctrica [31] que al mezclar con PEO y Sangre de drago en concentraciones bajas en agua destilada puede ser electrohilada. La solución acuosa de fibroína de seda debe ser almacenada a $4^{\circ} \mathrm{C}$ por el lapso de 30 días, antes de que esta se degrade por completo y no sea electrohilable.

\section{Electrospinning}

Una vez realizada la mezcla de fibroína de seda con PEO, se coloca la solución en una jeringa de $10[\mathrm{~mL}]$ y posteriormente en la bomba de inyección, se prepara el plato colector con papel aluminio o gasa, precautelando así la fácil extracción de la membrana al final del proceso y se lo coloca a una distancia entre 12 y 16 [cm] de la punta del capilar. Posteriormente, se conecta el polo positivo de la fuente de alto voltaje en el capilar y el polo negativo en el colector, se setea el caudal entre 0.5 y $1.5[\mathrm{~mL} / \mathrm{hr}]$ y se aplica un voltaje afín de generar un cono de Taylor estable entre 5 y $10[\mathrm{kV}]$.

\section{Caracterización}

Se realizó el análisis morfológico en un microscopio electrónico de barrido (SEM) del Laboratorio de Microscopia del CENCINAT y el análisis mecánico del módulo de Young en el microscopio AFM MFP-3D de la Universidad de Antioquia, Medellín, Colombia.

\section{ANÁLISIS DE RESUlTADOS}

Debido a problemas de solubilidad con la sangre de drago, que fueron estudiados experimentalmente con una serie de polímeros se llegó a la conclusión de que la savia únicamente genera mezclas uniformes con soluciones de bajo peso molecular, característica ajena a la solución de RSF+PEO. De esta forma fue posible únicamente mezclarlos cuando la sangre está diluida al 2\%v/v.

\section{A. Analisis Morfológico}

Se obtuvieron membranas de Fibroína de seda con PEO al $3 \% \mathrm{w} / \mathrm{v}$ con un diámetro medio de 139,43 nm y una desviación estándar de 19,26nm como se muestra en la Fig.1(a); así como membranas de fibroína de seda con PEO al $3 \% \mathrm{w} / \mathrm{v}$ y sangre de drago al $2 \% \mathrm{v} / \mathrm{v}$ como se muestra en la Fig. 1(b). Las dos muestras obtenidas se caracterizan por ser manipulables formadas de fibras continuas con presencia de beads porosos. Los beads porosos y la membrana que cuenta con interticios son unos de los principales requerimientos en apósitos para acelerar la migración celular y por ende el crecimiento de células epiteliales que regeneren el tejido. 




(a)

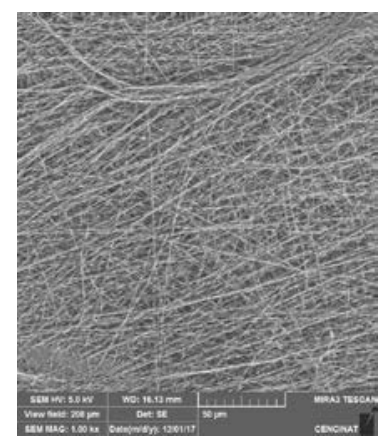

(b)
Fig. 1. Membranas de (a) RSF + PEO3\%w/v (b)RSF + PEO3\%w/v + SD $2 \% \mathrm{v} / \mathrm{v}$

\section{B. Análisis Mecánico de módulo de Young}

Para la muestra de RSF + PEO 3\% w/v se reportó un valor promedio de 279,9 [MPa] con desviación estándar de 167 [MPa]. La Fig. 2 presenta el histograma de frecuencia de los datos.

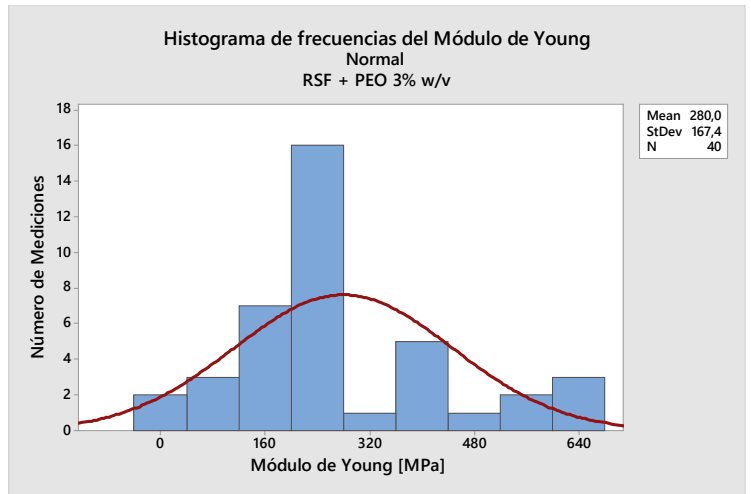

Fig. 2. Histograma de frecuencia del Módulo de Young (RSF + PEO $3 \% \mathrm{w} / \mathrm{v}$ )

Para la muestra de RSF + PEO 3\% w/v + SD 2\% v/v se reportó un valor promedio de 107,1 [MPa] con desviación estándar de 70,08 [MPa], lo que quiere decir que, aunque la adición de sangre de drago aumenta la viscosidad de la solución acuosa también disminuye el valor del módulo de Young de la membrana obtenida por electrospinning. La Fig. 3 presenta el histograma de frecuencia de los datos.



Fig. 3. Histograma de frecuencia del Módulo de Young (RSF + PEO 3\% $\mathrm{w} / \mathrm{v}+\mathrm{SD} 2 \% \mathrm{v} / \mathrm{v})$

En las figuras previamente presentadas se observa que no existe uniformidad en cuanto a los valores obtenidos, siendo la desviación estándar de los valores del Módulo de Young relativamente altos, esto producto del número reducido de pruebas realizadas dada la complejidad y por ende coste de las mismas, así como de la variación de los diámetros de las fibras cuya desviación estándar también tendía a ser alta.

\section{CONCLUSIONES}

Producto de la caracterización morfológica se obtuvieron las mejores muestras a los siguientes parámetros operacionales de electrospinning: Voltaje: 6-7 [kV], caudal: 1,2-1,4 [mL/h] y distancia entre el colector/capilar: $12[\mathrm{~cm}]$ y parámetros ambientales: Temperatura: $18-19{ }^{\circ} \mathrm{C}$ y humedad relativa: 60-63\% , tras el análisis mediante microscopía SEM se obtuvieron los siguientes datos, por un lado para la solución RSF + PEO $3 \%$ w/v se obtuvo diámetro medio de 139,43[nm] con una desviación estándar de 19,26[nm] siendo esta la mejor muestra en cuanto a morfología, mientras que la solución RSF + PEO 3\%w/v + $\mathrm{SD} 2 \% \mathrm{v} / \mathrm{v}$ tuvo un diámetro medio de $160[\mathrm{~nm}]$ con una desviación estándar de 47,14,19[nm] con presencia de beads porosos que tenían como diámetro medio de poro 30,19[nm] y desviación de 8,81[nm] siendo las dos membranas manipulables que pueden ser utilizadas como apósitos en heridas tras análisis biológicos. Los datos de Módulo de Young obtenidos de las pruebas realizadas en el Microscopio de Fuerza Atómica AFM muestra que las fibras en base a fibroína de seda RSF + PEO 3\% w/v tiene un valor promedio de 279,9 [MPa] con una desviación estándar de 167[MPa], mientras que al agregarle la savia de sangre de drago obteniendo la solución RSF + PEO 3\% w/v + SD 2\% $\mathrm{v} / \mathrm{v}$ tiene un valor promedio de 170,1 [MPa] con una desviación estándar de 70,08 [MPa]. Además, se puede concluir que los altos valores de desviación estándar en las pruebas demuestran que es necesario realizar más ensayos con variaciones en los diferentes parámetros, lo que se dificulta por la complejidad técnica del ensayo y el elevado costo de la misma, además de procurar que la desviación estándar del diámetro de las fibras ensayadas no tienda a ser muy elevado, para el caso en particular la desviación para la primera muestra es de 19,26 [nm], mientras que para el segundo caso es de 47,14 [nm] que en escala nanométrica es relativamente alto provocado por la dificultad presentada al trabajar con biopolímeros y el reducido control que se tiene sobre los parámetros ambientales en el proceso de electrospinning. Adicionar sangre de drago a la solución de RSF + PEO 3\% w/v a pesar de aumentar la viscosidad de la solución tiende a reducir el módulo de Young de las fibras obtenidas del proceso de electrospinning, aspecto que no cuenta con mucha relevancia para el caso de un apósito donde un parche secundario se caracteriza por su rigidez y resistencia mecánica mientras que el apósito primario como el elaborado, es el que cuenta con los agentes biológicos que aportan a acelerar la cicatrización de la herida.

\section{AGRADECIMIENTOS}

Este trabajo fue apoyado por el proyecto de investigación PIC-16-BENS-002 del programa becas ENSAMBLE de la SENESCYT. Agradecemos a nuestros colegas del Laboratorio de Reología y Fluidos Complejos y el Laboratorio de Microscopia del Centro de Nanociencia y Nanotecnología la Universidad de las Fuerzas Armadas ESPE. Del mismo modo, un agradecimiento al Dr. Salvador 
Aznár Cervantes por su colaboración activa en el desarrollo del estudio.

\section{REFERENCIAS}

[1] W. Li, E. Caterson, R. Tuan y F. Ko, «Electrospun Nanofibrous Structure a Novel Scaffold for Tissue Engineering,» J. Biomed. Mater. Res, pp. 60, 613, 2012.

[2] L. Niklason, «Engineering of bone grafts,» Nature Biotechnol. 18, pp. 929-930, 2000.

[3] M. S. Khil, D. Cha, H. Kim, I. Kim y N. Bhattari, «Electrospun nanofibrous polyurethane membrane as wound dressing,» Biomed. Res. 67B, pp. 675-679, 2003.

[4] N. Percival, "Classification of wounds and their management,» Surgery (Oxford), vol. 20, no 5, pp. 114-117, 2002.

[5] J. Venugopal, L. Ma y S. Ramakrishna, «Biocompatible nanofiber matrices for engineering dermal substitute for skin regeneration,» Tissue Engineering, 2005.

[6] S. R. Bhattarai, N. Bhattarai, H. Yi, P. Hwang, D. Cha y H. Kim, «Novel biodegradable electrospun membrane: scaffold for tissue engineering,» Biomaterials. 25, pp. 2595-2602, 2004.

[7] P. Zahedi, I. Rezaeian, S. O. Ranaei-Siadat, S. H. Jafari y P. Supaphol, «A review on wound dressings with an emphasis on electrospun nanofibrous polymeric bandages,» Polymers for Advanced Technologies 21, pp. 77-95, 2010.

[8] G. T. Lionelli y T. W. Lawrence, «Wound dressings,» Surg. Clin. North Am. 83, p. 617, 2003.

[9] J. J. Elsner y M. Zilberman, «Antibiotic-eluting bioresorbable composite fibers for wound healing applications: Microstructure, drug delivery and mechanical properties,» Acta Biomater.5, p. 2872-2883, 2009.

[10] E. Chevalier, D. Chulia, C. Pouget y M. Viana, «Fabrication of porous substrates: A review of processes using pore forming agents in the biomaterial field,» J. Pharm. Sci, p. 1135-1154, 2008.

[11] X. Xu, J. Zhang y Y. Fan, Biomacromolecules, pp. 2283-2289, 2010.

[12] L. A. Smith y P. X. Ma, «Nano-fibrous scaffolds for tissue engineering,» Colloids and Surfaces B: Biointerfaces, vol. 39, pp. 125-131, 2004

[13] Y. Zhou, D. Yang, X. Chen, Q. Xu, F. Lu y J. Nie, «Electrospun water-soluble carboxyethyl chitosan/poly(vinyl alcohol) nanofibrous membrane as potential wound dressing for skin regeneration,» Biomacromolecules, vol. 9, pp. 349-354, 2006.

[14] M. Calafat, G. Guinea, J. Rigueiro y G. Plaza, «Usos médicos de la seda,» Investigación y Ciencia, pp. 28-35, 2011.

[15] U. Kim, J. Park, H. Kim, M. Wada y D. Kaplan, «Three dimensional aqueous-derived biomaterial scaffolds from silk fibroin,» Biomaterials. 26, pp. 2775-2785, 2005.

[16] D. Gaviria y L. C. Caballero, «Uso de biomateriales a partir de la fibroína de la seda de gusano de seda (Bombyx mori L.) Para procesos de medicina regenerativa basada en ingeniería de tejidos,» Rev. Méd. Risaralda, pp. 38-43, 2015.

[17] Y. Wong, H. J. Kim, G. Vunjak-Novakovic y D. L. Kaplan, «Stem cell-based tissue engineering with silk biomaterials,» Biomaterials. 27, pp. 6064-6082, 2006.

[18] G. Altman, F. Diaz, C. Jakuba, T. Calabro, R. L. Horan, J. Chen, H. Lu, J. Richmond y D. L. Kaplan, «Silk-based biomaterials,» Biomaterials, 2003.

[19] S. H. Kim, Y. S. Nam, T. S. Lee y W. H. Park, «Silk fibroin nanofiber. Electrospinning, properties, and structure,» Polym. J. 35, pp. 185-190, 2003.

[20] J. Kundu, M. Dewan, S. Ghoshal y S. Kundu, «Mulberry nonengineered silk gland protein vis- à-vis silk cocoon protein engineered by silkworms as biomaterial matrices,» Mater. Sci. Mater. Med . 19, p. 2679-2689, 2008.

[21] S. Kundu, B. Kundu, S. Talukdar, S. Bano, S. Nayak, J. Kundu, B. Mandal, N. Bhardwaj, M. Botlagunta, B. Dash, C. Acharya y A. Ghosh, «Invited review nonmulberry silk biopolymers,» Biopolymers. 97, p. 455-467, 2012.

[22] L. Wray, X. Hu, J. Gallego, I. Georgakoudi, F. Omenetto, D. Schmidt y D. Kaplan, «Effect of processing on silk-based biomaterials: reproducibility and biocompatibility,» J Biomed Mater Res B Appl Biomater. 99, p. 89-101, 2011.

[23] D. Gupta, B. Bleakley y R. K. Gupta, «Dragon's blood: Botany, chemistry and therapeutic uses,» Journal of Ethnopharmacology, 115, p. 361-380, 2008.

[24] P. Allaica Tenesaca, Comparación del efecto cicatrizante de tinturas elaboradas a base de guarango (caesalpinia spinosa) y sangre de drago (croton lechleri) aplicados en ratones (mus musculus)., Riobamba: Tesis, 2015.
[25] A. Vaisber y J. Millan, Taspine in the Cicatrizant Principle in Sangre de Grado Extracte from (Croton lechlerii), Córdova. Argentina: Plantas M.C Vol. 5, 1989.

[26] J. Lobardo, Diccionario Médico, España: Ediciones Doyna. Pág. 400, 1994.

[27] A. Herforth, Antifungal plants of the Peruvian Amazon: A Survey of ethnomedicinal uses and biological activity, Cornell University USA: Honors Thesis, 2002.

[28] S. N. Elliot, A. Buret, W. McKnight, M. Miller y J. L. Wallace, «Bacteria rapidly colonize and delay the healing of gastric ulcers in rats,» Am. J Physiol Gastrointest Liver Physiol. 275, pp. 424-432, 1998. 